\title{
(2) OPEN ACCESS \\ Prediagnosis pathway benchmarking audit in patients with Duchenne muscular dystrophy
}

\author{
Vasantha Lakshmi Gowda (1) , ${ }^{1}$ Miguel Fernandez, ${ }^{1}$ Manish Prasad, ${ }^{2}$ \\ Anne-Marie Childs, ${ }^{3}$ Imelda Hughes, ${ }^{4}$ Sandya Tirupathi, ${ }^{5}$ \\ Christian Gaudentius Engelbert Lourens De Goede ${ }^{0},{ }^{6}$ Declan O'Rourke, \\ Deepak Parasuraman, ${ }^{8}$ Tracey Willis, ${ }^{9}$ Samira Saberian, ${ }^{10}$ Ian Davidson ${ }^{11}$
}

\begin{abstract}
- Additional supplemental material is published online only. To view, please visit the journal online (http://dx.doi. org/10.1136/archdischild2020-321451)
\end{abstract}

For numbered affiliations see end of article.

Correspondence to Dr Vasantha Lakshmi Gowda, Paediatric Neurosciences, Evelina London Children's Hospital, London, UK; vasantha.gowda@gstt.nhs.uk

Received 18 December 2020 Accepted 4 June 2021

\begin{abstract}
Objective To describe age and time at key stages in the Duchenne muscular dystrophy (DMD) prediagnosis pathway at selected centres to identify opportunities for service improvement.

Design A multicentre retrospective national audit. Setting Nine tertiary neuromuscular centres across the UK and Ireland. A prior single-centre UK audit of 20 patients with no DMD family history provided benchmark criteria.

Patients Patients with a definitive diagnosis of DMD documented within 3 years prior to December 2018 ( $n=122)$.
\end{abstract}

Main outcome measures Mean age (months) at four key stages in the DMD diagnostic pathway and mean time (months) of presentational and diagnostic delay, and time from first reported symptoms to definitive diagnosis. Type of symptoms was also recorded. Results Overall, mean age at definitive diagnosis, age at first engagement with healthcare professional (HCP) and age at first reported symptoms were $53.9 \pm 29.7$, $49.9 \pm 28.9$ and $36.4 \pm 26.8$ months, respectively. The presentational delay and time to diagnosis were 21.1 $( \pm 21.1)$ and $4.6( \pm 7.9)$ months, respectively. The mean time from first reported symptoms to definitive diagnosis was $24.2 \pm 20.9$. The percentages of patients with motor and/or non-motor symptoms recorded were $88 \%$ ( $n=106 / 121)$ and $47 \%(n=57 / 121)$, respectively.

Conclusions Majority of data mirrored the benchmark audit. However, while the time to diagnosis was shorter, a presentational delay was observed. Failure to recognise early symptoms of DMD could be a contributing factor and represents an unmet need in the diagnosis pathway. Methods determining how to improve this need to be explored.

\section{INTRODUCTION}

Duchenne muscular dystrophy (DMD) is an $\mathrm{X}$-linked progressive neuromuscular disease caused by mutations in the DMD gene that encodes dystrophin. ${ }^{1}$ Although DMD is often maternally inherited, approximately one-third of cases occur as a result of spontaneous mutation. ${ }^{1}$ DMD is the most common childhood form of muscular dystrophy and characteristic manifestations are progressive muscle weakness, cardiorespiratory impairment and premature death. ${ }^{1}$ In the UK, the incidence of DMD is estimated to be between 1.0 and 2.8 per 10000 live male births, ${ }^{2}$ representing a high socioeconomic

\section{Key messages}

What is already known on this topic?

- A diagnostic pathway and methods to identify Duchenne muscular dystrophy (DMD) exist; however, this diagnostic pathway has remained unchanged since 2010.

- A UK audit of 20 patients with no family history of DMD was conducted in 2014 and served as a benchmark.

- Diagnostic delay is a recurring theme and studies indicate a mean diagnosis age of approximately 5 years, which has remained relatively unchanged.

What this study adds?

- Age at DMD diagnosis remains unchanged irrespective of family history. However, diagnosis was reached quickly once the signs were recognised and child referred.

- A presentational delay was observed, which was twice of that reported in the benchmark audit. Parents of the patients recognised early symptoms of DMD.

- An unmet clinical need exists relating to the requirement for education into the early signs of DMD and developmental delay landmarks as means to reduce presentational delay.

and health-related quality of life burden. ${ }^{3}$ There is no curative treatment for DMD and no UK-wide DMD newborn screening programme. ${ }^{45}$ However, an early and accurate diagnosis of DMD is crucial to ensure proactive treatment. ${ }^{6}$

In most cases, a successful diagnosis of DMD can be made following the recommended diagnosis pathway outlined by the DMD Care Considerations Working Group diagnostic (DMD-CCWG). ${ }^{6}$ This pathway includes a review of the patient's clinical history, family history and physical examination with confirmatory investigations, including serum creatine kinase measurement, genetic testing and/ or muscle biopsy. ${ }^{6}$ The diagnostic process usually begins in early childhood as signs and symptoms (such as muscle weakness, clumsiness, difficulty climbing stairs or toe walking) are noticed by parents, carers or healthcare professionals (HCPs) ${ }^{6}$ Despite the presence of the 2010 recommended diagnostic pathway and clear criteria for the identification of disease (presence of pathological 
dystrophin gene mutation), diagnostic delay is a recurring issue. ${ }^{7}$ Global population-based studies indicate a mean diagnosis age of approximately 5 years, ${ }^{8-13}$ which has remained relatively unchanged. ${ }^{8}$ However, a European study reported that the mean age of diagnosis in Europe had decreased to less than 3 years of age. ${ }^{14}$ Evidence suggests that socioeconomic status disparities, genotype and inherent issues with creatine kinase testing could be contributing to diagnostic delay. ${ }^{6} 8$

To identify opportunities for service improvement, a national real-world audit describing the time to DMD diagnosis and the diagnostic pathway of DMD was conducted in nine neuromuscular centres in the UK and Ireland. The audit outcome measures were benchmarked with reference to the recommended DMD diagnostic pathway ${ }^{6}$ and the ages at the different stages of the diagnostic pathway reported in a previous UK single-centre retrospective audit by van Ruiten et al, ${ }^{7}$ which included 20 boys without a family history of DMD. A clinical audit is a recognised strategy for improving outcomes in patients with DMD. ${ }^{15}$

\section{METHODS}

\section{Study design and patient population}

A multicentre audit of the DMD diagnostic pathway involving retrospective data collection from hospital medical records was conducted at nine neuromuscular centres across the UK and Ireland. Patients were included in the audit if they had a definitive diagnosis of DMD documented 3 years prior to December 2018. Eligible patients were identified, and data were collected by the direct care team. For the purposes of this audit, a definitive diagnosis of DMD was based on either a documented dystrophin mutation identified by genetic test/sequencing or a documented dystrophin-negative muscle biopsy, in instances where no genetic test/sequencing was documented. Patients recorded as having probable, possible or asymptomatic DMD were excluded.

\section{Audit endpoints and benchmarking criteria}

The diagnostic pathway recommended by the DMD Care Considerations Working Group provided a foundation for data collection relating to the different stages involved in establishing a $\mathrm{DMD}^{6}$ diagnosis. The benchmark criteria used in this audit for each stage of the diagnostic pathway are based on the van Ruiten et al study ${ }^{7}$ as outlined in table 1 . A complete list of the collected data is shown in online supplemental table 1 . As this was an audit and required no direct involvement with patients and no access to identifiable records by individuals outside the normal clinical care team, an application for research ethics committee approval was not necessary. Trust level approval was obtained from each participating Trust prior to patient enrolment.

\section{Data analysis}

All analyses were descriptive. Quantitative variables were summarised as mean, SD, median, IQR and range. Analyses were conducted for the overall patient population, and for stratified subgroups where appropriate. Data are presented as mean \pm SD unless otherwise stated, and percentages rounded to the nearest whole number (and as such may not sum to $100 \%$ ). In cases where data were incomplete, the denominator is stated. A single patient was diagnosed antenatally and was excluded from select analyses; these are highlighted in online supplemental table 1 .

Where partial data were provided, resulting in the time between two observations yielding a negative value $(n=7)$, the value on the partial data was assumed to be zero.

In the benchmark study, only patients without a family history of DMD were included ${ }^{7}$; therefore, in the present audit, analyses were stratified according to the presence/ absence of a documented family history of DMD. Two further subgroups relating to definitive diagnosis were created to examine extremities in the diagnostic ages. While no official definitions for early or late definitive diagnoses exist, for the purpose of this audit, early diagnosis was defined as a documented definitive diagnosis at age $<4$ years ( $<48$ months) and late diagnosis was defined as a definitive documented diagnosis at age $>5$ years ( $>60$ months).

\section{RESULTS}

A total of 122 eligible patients were included and the number of patients at participating centres ranged from 5 to 28 (a full list of participating centres is shown in online supplemental table 2). A family history of DMD was recorded for 26 out of 122 (21\%) patients; of these, 20 (77\%) had an affected first-degree

\section{Table 1 Benchmark criteria for each stage of the diagnostic pathway}

\begin{tabular}{|c|c|c|c|}
\hline Number & Recommendation $^{6}$ & Audit criteria & Benchmark standard $^{7}$ \\
\hline (1) & $\begin{array}{l}\text { DMD is confirmed as a definitive diagnosis once the presence of a } \\
\text { genetic mutation in the dystrophin gene has been established via } \\
\text { genetic testing or sequencing }\end{array}$ & Age at definitive diagnosis & Mean age: 51.7 months $^{7}$ \\
\hline (2) & $\begin{array}{l}\text { DMD should be suspected in a patient not walking by } 16-18 \\
\text { months, showing Gowers' sign, or toe walking (any age, especially } \\
<5 \text { years old) }{ }^{6}\end{array}$ & $\begin{array}{l}\text { Age at first engagement with a healthcare professional related to } \\
\text { reported symptoms suggestive of DMD }\end{array}$ & Mean age: 42.9 months $^{7}$ \\
\hline
\end{tabular}

Age at first reported symptoms, defined as age of first reported Mean age: 32.5 months ${ }^{7}$ concerns by parents

(4) CK test should be performed if unexplained increase in transaminases or the patient has symptoms suggestive of $\mathrm{DMD}^{6}$

(5) In the absence of neonatal screening for DMD, parents need to initiate contact with a healthcare professional if they, or persons involved in caring for their child (eg, nursery teachers), have concerns regarding the development of their child

(6) Healthcare professionals should follow guidelines to establish the diagnosis of DMD. ${ }^{6}$ Rapid referral to a neuromuscular specialist, with input from a geneticist or genetic counsellor is recommended. ${ }^{9}$
Age at first blood test result with measurement of CK or CK-MM

Mean age: 50.1 months $^{7}$

Time from first reported symptoms to first engagement with a Mean time: 10.4 months healthcare professional (presentational delay), defined as time from (3) to (2)

Time from first engagement with a healthcare professional to date Mean time: 8.8 months $^{7}$ of definitive diagnosis (diagnostic delay), defined as time from (2) to (1)

Total time from first reported symptoms to definitive diagnosis, defined as time from (3) to (1)

CK, creatine kinase; CK-MM, muscle creatine kinase; DMD, Duchenne muscular dystrophy. 
A

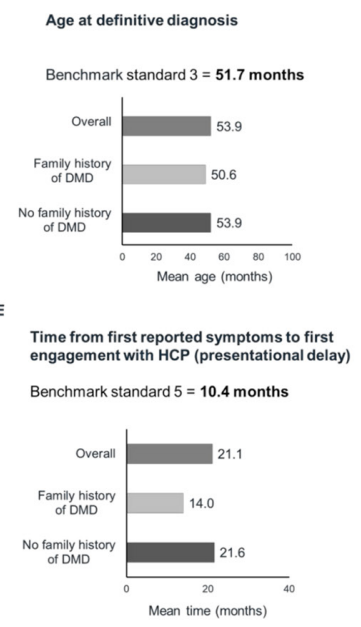

B

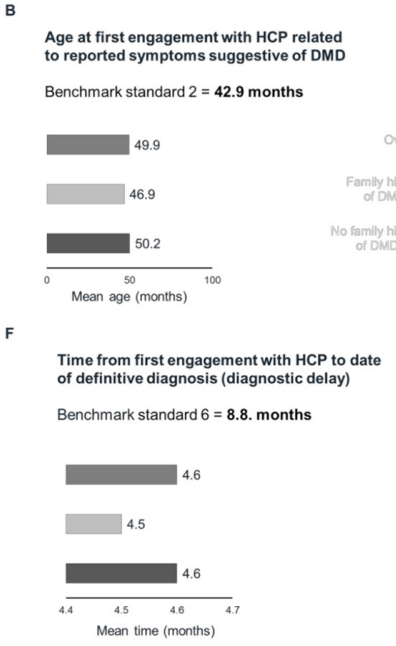

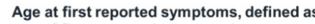
age of first reported concerns by parents Benchmark standard $1=32.5$ months

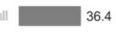

40.7

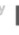

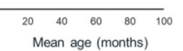

G

Total time from first reported symptoms to definitive diagnosis

Benchmark standard $7=19.2$ months

24.2

$\square 15.1$

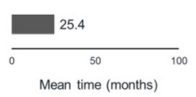

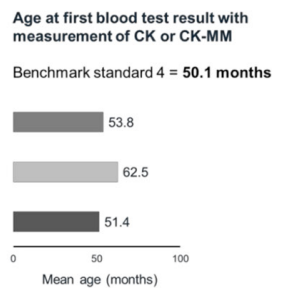

Age at first blood test result with
measurement of CK or CK-MM

Benchmark standard $4=50.1$ months

CK. creatine kinase: CK-MM, muscle creatine kinase: DMD, Duchenne muscular dystrophy; HCP, heatthcare professiona

Figure 1 Age and diagnostic time periods at key stages in the DMD diagnostic pathway overall and stratified by DMD family history. Mean age (months) and time (months) at key stages in the diagnostic pathway are presented for the overall population and stratified by DMD family history (A-G). CK, creatine kinase; CK-MM, muscle creatine kinase; DMD, Duchenne muscular dystrophy; HCP, healthcare professional.

relative, $4(15 \%)$ had an affected second-degree relative, $1(4 \%)$ had an affected distant relative and familial relationship was not recorded for 1 (4\%). Of the patients with a postnatal diagnosis of DMD, 51 out of 121 (42\%) were <48 months, 27 out of 121 (22\%) were 48-60 months and 42 out of $121(35 \%)$ were $>60$ months of age at diagnosis.

\section{Assessment of age and diagnostic time periods at key stages in the DMD diagnostic pathway}

Results for age and diagnostic time periods at key stages in the DMD diagnostic pathway are shown in figure 1 and summary statistics in online supplemental tables 3,4. The mean age at definitive diagnosis in the overall population $(\mathrm{n}=120)$ was $53.9( \pm 29.7)$ months (family history: 50.6 $( \pm 44.1)$ months, $(\mathrm{n}=25)$; no family history $53.9( \pm 24.6)$ months, $(\mathrm{n}=92)$ ) (figure 1A). The median (IQR) age at definitive diagnosis in the overall population $(\mathrm{n}=120)$ was 52.2 (31.5-65.0) months (family history: 36.9 (12.8-64.9) months, $(\mathrm{n}=25)$; no family history 52.4 (37.9-64.0) months, $(\mathrm{n}=92))$ (online supplemental table 3$)$.

The mean ages (months) at first engagement with HCP, age at first reported symptoms, age at first blood test result in the overall population were $49.9 \pm 28.9 \quad(n=106), \quad 36.4 \pm 26.8$ $(\mathrm{n}=66)$ and $53.8 \pm 30.1(\mathrm{n}=97)$, respectively. Data relating to patients with an early diagnosis or late diagnosis are summarised in online supplemental table 4.

In relation to presentational delay and time to diagnosis, the mean times (months) for the overall population for first reported symptoms to first HCP engagement, first engagement with HCP to date of definitive diagnosis and from first reported symptoms to definitive diagnosis were $21.1 \pm 21.1(\mathrm{n}=59), 4.6 \pm 7.9$ $(\mathrm{n}=106)$ and $24.2 \pm 20.9(\mathrm{n}=66)$, respectively (figure $1 \mathrm{E}-\mathrm{G})$. Summary statistics in relation to patients with and without a family history are shown in online supplemental table 3 . For patients with an early diagnosis and late diagnosis, data relating to age and time at key stages of the diagnostic pathway are summarised in online supplemental figure 1 and online supplemental table 4.

\section{Symptoms of DMD}

The percentage of patients with motor and non-motor symptoms and the types of symptoms experienced are summarised in figure $2 \mathrm{~A}, \mathrm{~B}$. The percentage of patients with motor and non-motor symptoms recorded in the overall population was $88 \%(106 / 121)$ and $47 \%(57 / 121)$, respectively (not mutually exclusive). No motor symptoms were recorded in 2 of the 57 patients who had non-motor symptoms recorded. Motor symptoms included gross motor delay and Gower's sign on rising from the floor, while non-motor symptoms included speech delay or articulation details and cognitive delay (figure $2 \mathrm{~B}$ ). In the majority of cases (overall population: $73 \%(77 / 106),(n=106))$, it was the parent who noticed the early motor symptoms of DMD (online supplemental table 5). Motor and non-motor symptoms experienced by patients classified as early diagnosis and late diagnosis are summarised in online supplemental figure 2 .

\section{Diagnostic tests performed}

Overall, $100 \%(121 / 121)$ of patients received a creatine kinase test (CKT) (figure 3). The distribution of HCPs requesting CKTs for the overall population and in patients with and without a family history is summarised in online supplemental table 7. Overall, 97\% (118/122) of patients underwent a genetic test for DMD (DMD gene deletion or duplication) (family history: $92 \%$ (24/26); no family history: $98 \%(91 / 93))$ and $20 \%(24 / 122)$ of patients had DMD genetic sequencing (family history: 12\% (3/26); no family history: $23 \%(21 / 93))$. In patients diagnosed early and late, the diagnostic tests performed and the distribution of HCPs requesting a $\mathrm{CK}$ test are summarised in online supplemental figure 3 and online supplemental table 8 , respectively.

\section{Protocols followed to establish diagnosis of DMD}

The most commonly reported protocols used for establishing the diagnosis of DMD (see online supplemental table 9) were the following: guidance on https://childmuscleweakness.org/ ${ }^{16}(44 \%,[15 / 34])$; the integrated diagnostic pathway $(41 \%$, [14/34]); and Standards of Care for Duchenne muscular dystrophy $(29 \%,[10 / 34])$. Data in relation to patients with 
A

Overall $(n=121)^{*}$

a Yes not provided

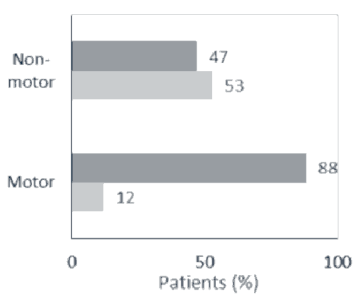

Motor symptoms*

B

B

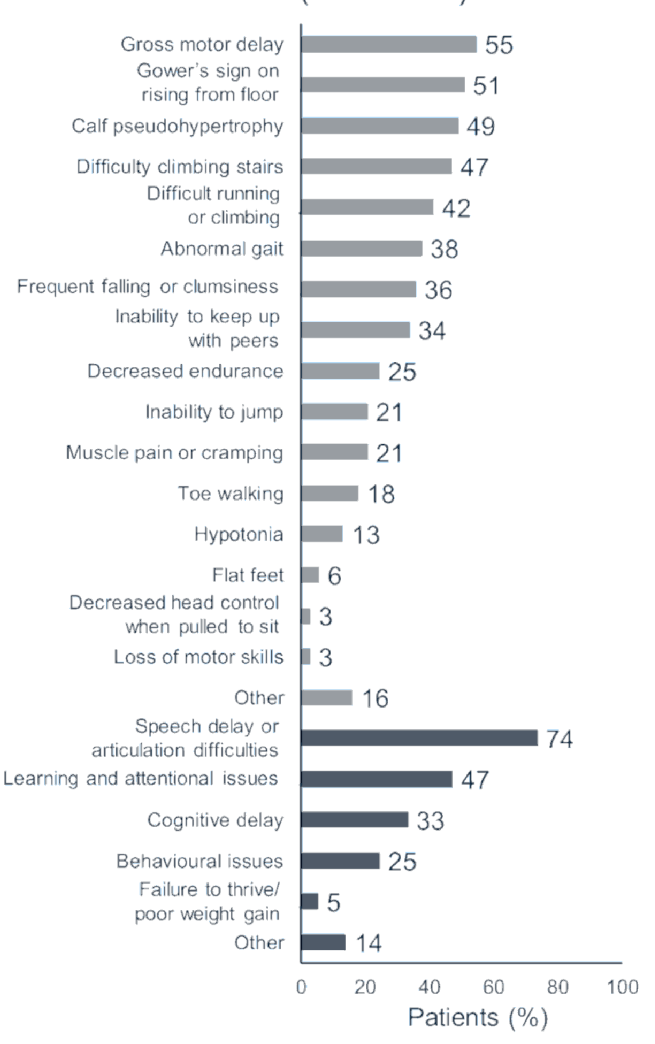

Overall

$(\mathrm{N}=106 / 57)$

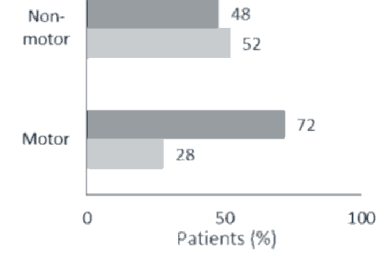

$(n=25)$

- Non-motor symptoms*

Family history

of DMD ( $n=18 / 12)$

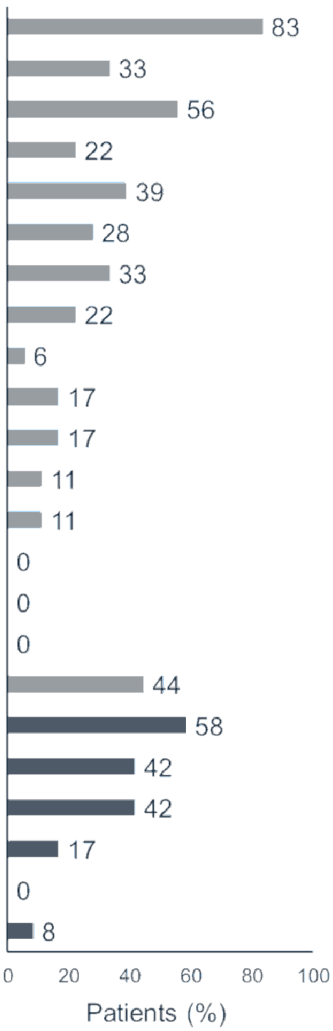

No family history

of DMD $(n=85 / 42)$

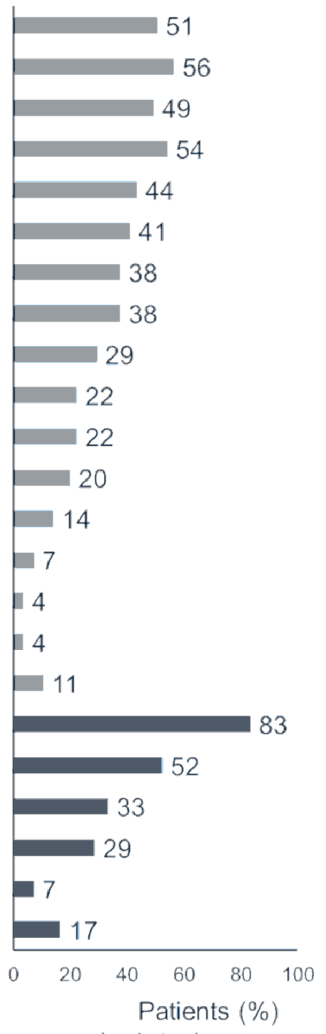

Figure 2 Symptoms presented by patients overall and stratified by DMD family history. The percentage of patients with motor and non-motor symptoms are presented for the overall population and stratified by DMD family history (A); the type of symptoms experienced in the overall patient population and by subgroup are also presented (B). * Not mutually exclusive because patients may have presented with $>1$ symptom. DMD, Duchenne muscular dystrophy.

an early diagnosis or late diagnosis are summarised in online supplemental table 10 .

\section{DISCUSSION}

An early and accurate diagnosis of DMD is crucial, as this allows for early treatment, early implementation of the standards of care (including drug therapy in subgroups where indicated) and early access to appropriate genetic counselling. ${ }^{17}$ The aims of this audit were to evaluate the time to diagnosis and to describe the prediagnosis pathway in patients diagnosed with DMD at selected centres in the UK and Ireland, with benchmarking against a previously published audit. ${ }^{7}$ The results of this audit were broadly similar to the previously published benchmark audit; however, several important distinctions were ascertained, which could have implications for the diagnostic pathway of DMD.

The mean age of patients at the time of definitive diagnosis for the overall population was 53.9 months, which is broadly similar to the benchmark audit (51.7 months $)^{7}$ and a cross-sectional survey conducted in seven European countries (51 months). ${ }^{14}$ Results from the present audit suggest there was a slight change in the age at DMD diagnosis.

In this audit, the total time from the first reported symptoms to the time of definitive diagnosis was broadly similar to benchmark audit (19.2 months). ${ }^{7}$ However, the time from first engagement with HCP to definitive diagnosis was approximately half the time described in the benchmark audit ( 8.8 months). ${ }^{7}$ This demonstrates that once the signs are recognised and the child is 
- Liver testing* $\quad$ Genetic testing*

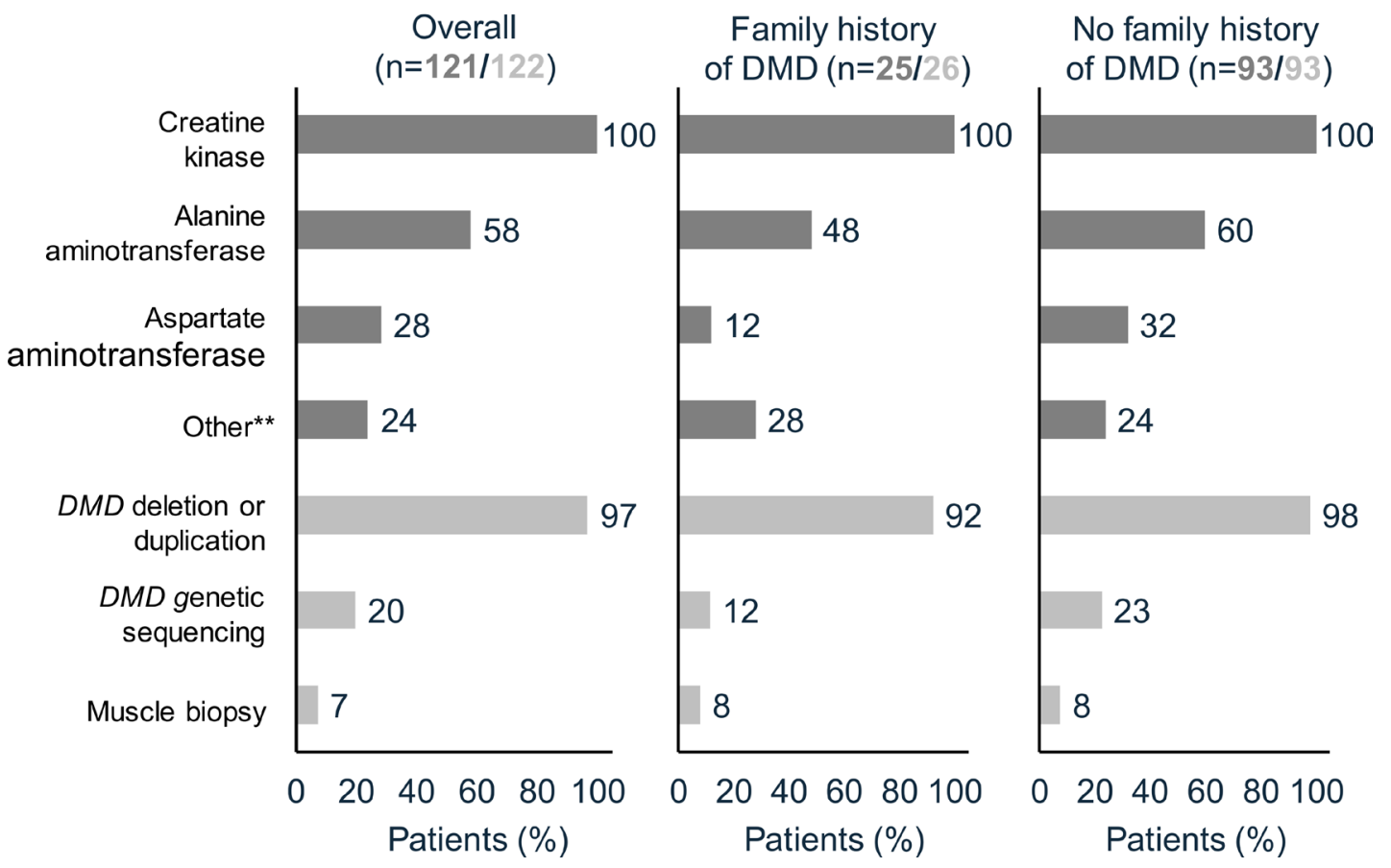

Figure 3 Diagnostic tests performed overall and stratified by DMD family history. The percentage of patients in whom diagnostic tests were performed are presented for the overall population and stratified by DMD family history. * Not mutually exclusive because patients may have presented with $>1$ symptom. ** Includes albumin, alkaline phosphatase, bilirubin, gamma-glutamyl transferase and vitamin D. DMD, Duchenne muscular dystrophy.

referred appropriately, a diagnosis is reached quickly. However, given that the general practitioner (GP) attendance record of a patient, as contained in their secondary care medical notes, may not be comprehensive, the patient's earliest engagement with a GP (HCP) may not have been captured. As such the true time from first engagement with HCP to definitive diagnosis may be longer than the observed 8.8 months.

Generally, genetic testing takes 1 month for deletion/duplication testing and if negative, an additional 3 months is required for acquisition of the full sequence. Collectively, these results suggest that the diagnostic delays highlighted in the earlier 2014 audit have perpetuated to this audit and appear to be mostly related to delays in initial recognition of symptoms or referral to a paediatrician.

The results of the present audit highlight a presentational delay, with the time from reported symptoms to first documented HCP visit approximately twice as long as reported in the benchmark audit (10.4 months). ${ }^{7}$ The potential reasons for the observed presentational delay may be due to social factors and the difficulty, by some parents for instance, ${ }^{7}$ in recognising the manifestations of DMD; these typically include motor symptoms (eg, motor milestone delay, inability to run/other gait signs, calf pseudohypertrophy and Gowers' sign) and non-motor symptoms (eg, speech or global developmental delay, behavioural and learning challenges, failure to thrive and fatigue). Almost half of patients in the audit had experienced non-motor symptoms prior to diagnosis and evidence suggests that failure to recognise nonmotor symptoms as a common feature of DMD can contribute to late diagnosis. ${ }^{11}$ These findings highlight a potential unmet need in the community for educational initiatives to raise awareness of the signs and symptoms of DMD. Such educational initiatives would need to be tailored accordingly for different groups in the community (eg, parents/carers, physiotherapists and paediatric nurses). Such initiatives may include the e-learning module launched by the Royal College of Paediatrics and Child Health to support early recognition of neuromuscular disorders. ${ }^{18}$ It is important to note that the impact of this e-learning may not be reflected in the findings of this audit.

To improve overall service, several opportunities could be explored. The presentational delay observed could be related to a lack of overall awareness of DMD in the community (HCPs and the general public), leading to failure to recognise the early signs and symptoms of DMD. Maximising the recognition of early symptoms of DMD through education of community teams, health visitors and nursery staff is crucial. Therefore, it may be useful to survey primary care HCPs to understand the most effective approach (eg, specific guidance) to encourage their routine assessment of motor and non-motor symptoms, particularly where there is evidence of speech or global developmental delay. A presentational delay means that patients do not get prompt referral to specialist centres to receive treatments, such as mutation-specific therapies. ${ }^{17}$

Any retrospective analysis of medical records will be limited by the quality and completeness of the available records. This is likely to have had its most pronounced impact on data related to contacts with HCPs. The number of HCP contacts may have been under-reported, given that GP attendance details may not be comprehensively documented in secondary care patient records. Additionally, the description of time from first reported symptom to first engagement with a healthcare professional was based on age at first reported concern, specifically by parents. As this age/date was not available for many patients, description 
of presentational delay was not based on all recruited participants. A future study that includes primary care data would be important to provide a comprehensive overview of the diagnostic pathway.

\section{CONCLUSION}

Many of the findings of this audit were in line with those from a previously published single-centre audit conducted in 2014, except for the time from first HCP engagement to definitive diagnosis and time from first reported symptoms to engagement with an HCP. There may still be delays from symptom onset to the first engagement with an HCP and subsequent referral for a definitive diagnosis. As there are more treatment options for DMD available today compared with 2014, there is an urgent need to reduce any presentational delay. Failure to recognise the early symptoms of DMD could be contributing to the observed presentational delay. As a result of this, patients will receive treatment later in disease progression, where certain treatments, such as gene-specific treatments, are more successful if initiated early. ${ }^{14}$ Ultimately, failure to recognise early DMD symptoms is an unmet need in the diagnosis pathway and additional strategies for improvements should be explored.

\section{Author affiliations}

'Paediatric Neurosciences, Evelina London Children's Hospital, London, UK

2Paediatrics, Queen's Medical Centre, Nottingham, UK

${ }^{3}$ Paediatric Neurology, Leeds Teaching Hospitals NHS Trust, Leeds, UK

${ }^{4}$ Paediatric Neurology, Royal Manchester Children's Hospital, Manchester University NHS Foundation Trust, Manchester, UK

${ }^{5}$ Paediatric Neurology, Royal Belfast Hospital for Sick Children, Belfast, UK

${ }^{6}$ Paediatric Neurology, Royal Preston Hospital, Preston, UK

${ }^{7}$ Neurology, Children's Health Ireland at Temple Street, Dublin, Ireland

${ }^{8}$ Paediatrics, University Hospitals Birmingham NHS Foundation Trust, Birmingham, UK

${ }^{9}$ Muscle Team, Robert Jones and Agnes Hunt Orthopaedic Hospital NHS Foundation

Trust, Oswestry, UK

${ }^{10}$ Evidence and Access, OPEN HEALTH, Marlow, UK

${ }^{11}$ UK \& Ireland Commercial Department, PTC Therapeutics Limited, Guildford, UK

\section{Twitter Vasantha Lakshmi Gowda @vasanthagowda}

Acknowledgements The authors thank OPEN VIE, a healthcare consultancy company specialising in real-world evidence, who were commissioned by PTC therapeutics to provide support for audit design and implementation, data analysis and interpretation, and medical writing support. Editorial support for development of this manuscript was provided by Sarah Stowell and Edward Ottley from OPEN VIE in accordance with Good Publication Practice 3 guidance, funded by PTC Therapeutics.

Contributors VLG, MP, AMC, IH, ST, CGELDG, DOR, DP, TW and ID were involved in the conception and design of the study. SS analysed the study data. All authors were involved in the interpretation of the data for the work, critical revision of the manuscript and, read and approved the final version of the manuscript. All authors agree to be accountable for all aspects of the work.

Funding This work was funded by PTC Therapeutics.

Competing interests VLG has received grants from Biogen, PTC Therapeutics, Wave Life Sciences and Catabasis; MF has nothing to disclose; MP has received a grant from PTC Therapeutics; AMC has received fees from PTC Therapeutics in relation to contributions to advisory boards, invited lectures and a presentation; IH has nothing to declare; ST has received conference sponsorship; CGELDG has received grants from PTC Therapeutics; DOR has nothing to declare; DP has nothing to declare; TW has received honorariums for lectures and advisory boards from PTC therapeutics, Biogen, Genzyme Sanofi, Roche, Sarepta and Santhera; SS is an employee of OPEN VIE; ID is employed by PTC Therapeutics.

Patient consent for publication Not required.

Provenance and peer review Not commissioned; externally peer reviewed.

Data availability statement No data are available. As this was a service audit that did not require patient consent, we are unable to provide access to the data.
Supplemental material This content has been supplied by the author(s). It has not been vetted by BMJ Publishing Group Limited (BMJ) and may not have been peer-reviewed. Any opinions or recommendations discussed are solely those of the author(s) and are not endorsed by BMJ. BMJ disclaims all liability and responsibility arising from any reliance placed on the content. Where the content includes any translated material, BMJ does not warrant the accuracy and reliability of the translations (including but not limited to local regulations, clinical guidelines, terminology, drug names and drug dosages), and is not responsible for any error and/or omissions arising from translation and adaptation or otherwise.

Open access This is an open access article distributed in accordance with the Creative Commons Attribution Non Commercial (CC BY-NC 4.0) license, which permits others to distribute, remix, adapt, build upon this work non-commercially, and license their derivative works on different terms, provided the original work is properly cited, appropriate credit is given, any changes made indicated, and the use is non-commercial. See: http://creativecommons.org/licenses/by-nc/4.0/.

\section{ORCID iDs}

Vasantha Lakshmi Gowda http://orcid.org/0000-0002-8367-5489

Christian Gaudentius Engelbert Lourens De Goede http://orcid.org/0000-0001-77725972

\section{REFERENCES}

1 Mah JK. Current and emerging treatment strategies for Duchenne muscular dystrophy. Neuropsychiatr Dis Treat 2016;12:1795-807.

2 Mah JK, Korngut L, Dykeman J, et al. A systematic review and meta-analysis on the epidemiology of Duchenne and Becker muscular dystrophy. Neuromuscul Disord 2014:24:482-91.

3 Cavazza M, Kodra Y, Armeni P, et al. Social/economic costs and health-related quality of life in patients with Duchenne muscular dystrophy in Europe. Eur J Health Econ 2016;17 Suppl 1:19-29.

4 Moat SJ, Bradley DM, Salmon R, et al. Newborn bloodspot screening for Duchenne muscular dystrophy: 21 years experience in Wales (UK). Eur I Hum Genet 2013;21:1049-53.

5 nhs.uk. Newborn blood spot test [Internet], 2017. Available: https://www.nhs.uk/ conditions/pregnancy-and-baby/newborn-blood-spot-test/ [Accessed 06 Jul 2020].

6 Birnkrant DJ, Bushby K, Bann CM, et al. Diagnosis and management of Duchenne muscular dystrophy, part 1: diagnosis, and neuromuscular, rehabilitation, endocrine, and gastrointestinal and nutritional management. Lancet Neurol 2018;17:251-67.

7 van Ruiten HJA, Straub V, Bushby K, et al. Improving recognition of Duchenne muscular dystrophy: a retrospective case note review. Arch Dis Child 2014:99:1074-7.

8 Counterman KJ, Furlong P, Wang RT, et al. Delays in diagnosis of Duchenne muscular dystrophy: an evaluation of genotypic and sociodemographic factors. Muscle Nerve 2020;61:36-43.

9 Ciafaloni E, Fox DJ, Pandya S, et al. Delayed diagnosis in Duchenne muscular dystrophy: data from the muscular dystrophy surveillance, tracking, and research network (MD STARnet).J Pediatr 2009:155:380-5.

10 D'Amico A, Catteruccia M, Baranello G, et al. Diagnosis of Duchenne muscular dystrophy in Italy in the last decade: critical issues and areas for improvements. Neuromuscul Disord 2017;27:447-51

11 Mohamed K, Appleton R, Nicolaides P. Delayed diagnosis of Duchenne muscular dystrophy. Eur J Paediatr Neurol 2000;4:219-23.

12 Wong SH, McClaren BJ, Archibald AD, et al. A mixed methods study of age at diagnosis and diagnostic odyssey for Duchenne muscular dystrophy. Eur I Hum Genet 2015;23:1294-300

13 Holtzer C, Meaney FJ, Andrews J, et al. Disparities in the diagnostic process of Duchenne and Becker muscular dystrophy. Genet Med 2011;13:942-7.

14 Vry J, Gramsch K, Rodger S, et al. European Cross-Sectional Survey of Current Care Practices for Duchenne Muscular Dystrophy Reveals Regional and Age-Dependent Differences. J Neuromuscul Dis 2016:3:517-27.

15 HQIP. Documenting local clinical audit - a guide to reporting and recording [Internet], 2016. Available: https://www.haip.org.uk/resource/documenting-local-clinical-audita-guide-to-reporting-and-recording/ [Accessed 07 Jul 2020].

16 Child Muscle Weakness. Home [Internet]. Available: https://childmuscleweakness.org/ [Accessed 08 Jun 2020].

17 Aartsma-Rus A, Hegde M, Ben-Omran T, et al. Evidence-Based consensus and systematic review on reducing the time to diagnosis of Duchenne muscular dystrophy. J Pediatr 2019;204:305-13.

18 Action Duchenne. Recognising Neuromuscular Disorders eLearning module is now live [Internet], 2018. Available: https://www.actionduchenne.org/recognisingneuromuscular-disorders-elearning-module-is-now-live/ [Accessed 04 Feb 2021]. 